\title{
A window of opportunity
}

DOI:

10.1038/nrn2178

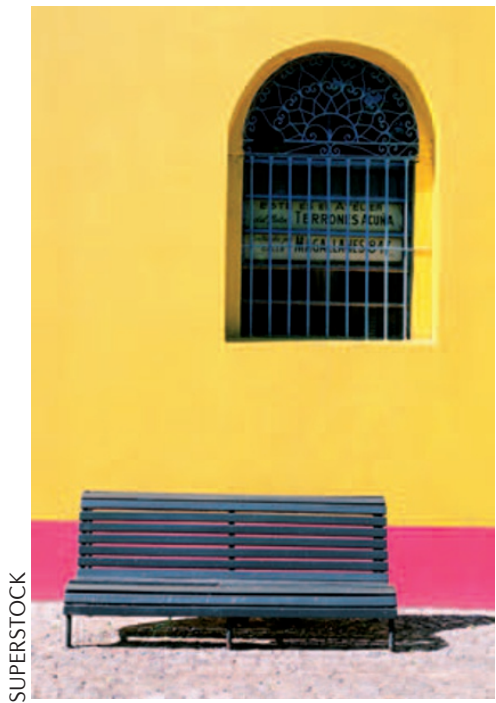

The existence of adult hippocampal neurogenesis is clearly established, yet it remains unclear whether adult-born neurons make a specific contribution to hippocampal function or merely act as a reservoir to replace dying neurons. Song and colleagues now demonstrate a transient 'critical period' of enhanced synaptic plasticity in these neurons, suggesting that the ongoing birth of neurons may help to maintain plasticity in the adult hippocampus.

During development, newlyformed synapses are particularly susceptible to modification by experience-driven activity during a defined critical period. In the adult, memory formation is likely to involve modifications of hippocampal circuitry, yet it is also essential that the established circuitry remains stable. This raises the question of whether new-born cells might be important for such plasticity. To determine whether these cells are more susceptible to synaptic modification than their more mature counterparts, the authors labelled adult-born dentate gyrus cells (DGCs) in mice using a GFP-expressing retrovirus, and compared their electrophysiological properties with those of mature DGCs.

Using this technique, the authors were able to construct an accurate time course of synaptic properties in adult-born neurons as they develop. They identified a window between 1 and 1.5 months after the neurons' birth during which the neurons exhibited enhanced synaptic plasticity, including a decrease in the threshold for long-term potentiation (LTP) induction and an increase in LTP amplitude.

LTP requires the activity of NMDA ( $N$-methyl-D-aspartate) receptors, which are composed of NR1 and NR2 subunits. The NR2B subunit is expressed at nascent synapses and is linked to enhanced plasticity during developmental critical periods. The authors therefore examined the effects of ifenprodil, a specific blocker of NR2B-containing
NMDARs, on the synaptic plasticity of DGCs. Ifenprodil dramatically reduced LTP in DGCs that were between 1 and 1.5 months old, but had a much smaller effect on DGCs outside this 'critical period'. Furthermore, blocking 25\% of NMDA-mediated activity using ifenprodil had a much larger impact on LTP in these neurons than the same degree of NMDA-blockade using the pan-NMDAR blocker APV, suggesting that NR2B is essential for synaptic plasticity in adult-born DGCs during the critical period and that it cannot be replaced with other NMDAR subtypes.

This study reveals the existence of a critical period of synaptic plasticity in adult-born hippocampal neurons, analogous to that observed in developing neural circuits. It also shows that the NR2B receptor subunit recapitulates its developmental role in establishing this enhanced plasticity. This could point to a unique contribution of these cells to experience-induced plasticity in the adult hippocampus, although further work will be required to establish such a function.

Katherine Whalley

ORIGINAL RESEARCH PAPER Ge, S., Yang, C. H., Hsu, K. S., Ming, G. L. \& Song, H. A critical period for enhanced synaptic plasticity in newly generated neurons of the adult brain. Neuron 54 559-566 (2007) 